\title{
Nanotechnology bring a new hope for asthmatics
}

\author{
Wen-Chien Cheng ${ }^{1}$, Chia-Hung Chen ${ }^{2}$ \\ ${ }^{1}$ Division of Pulmonary and Critical Care Medicine, Department of Internal Medicine, ${ }^{2}$ School of Medicine, China Medical University, Taichung \\ Correspondence to: Chia-Hung Chen, MD. Department of Internal Medicine, China Medical University Hospital, No. 2, Yude Road, Taichung 404. \\ Email: hsnu758@gmail.com. \\ Provenance: This is an invited article commissioned by the Editorial Office, Annals of Translational Medicine. \\ Comment on: Wang L, Feng M, Li Q, et al. Advances in nanotechnology and asthma. Ann Transl Med 2019;7:180.
}

Submitted Sep 02, 2019. Accepted for publication Sep 26, 2019.

doi: 10.21037/atm.2019.09.153

View this article at: http://dx.doi.org/10.21037/atm.2019.09.153

Asthma - a chronic inflammatory lung disease-is one of the most common major global health problems. Main characteristics of asthma encompass reversible airway obstruction and bronchial hyper-responsiveness (1). The Global Initiative for Asthma (GINA) guideline for the management of asthma includes the following distinct goals: alleviate and control symptoms, reduce asthma exacerbation, prevent the loss of pulmonary function, and minimize side effects. The most widely used asthma therapies are inhaled corticosteroids (ICSs) and bronchodilators, such as short-acting $\beta$-adrenergic agonists, long-acting $\beta$-adrenergic agonists (LABAs), muscarinic antagonists, and leukotriene receptor antagonists (2). However, the control of asthma is suboptimal in some asthmatic patients, even at GINA guideline step 4 or 5 (medium-to-high dose of ICSs/LABAs). This might be because asthma is characterized by heterogeneity and variability, in which genetic polymorphisms interact with environmental factors (3). ICSs might not provide immediate relief from asthma symptoms and might cause long-term side effects (4), which are associated with a decreased rate of treatment adherence (5). Decreased treatment adherence can accelerate the progression of the disease as well as lung remodeling, leading to progressive pulmonary deterioration (6). Additionally, long-term use of ICSs might lead to lung infection with opportunistic microorganisms owing to local pulmonary immunosuppression (7). Therefore, alternative therapies that can reduce inflammation and remodeling processes in asthma patients and relieve airway obstruction without causing immunosuppression for asthma control are needed.

Nanomedicine is a multidisciplinary field of research with integration of traditional sciences, including biology, ecology, chemistry, geology, engineering, and physics. The application of nanomedicine for lung diseases, as a relatively new area of interdisciplinary science, has grown rapidly over the last 10 years, particularly in the management of cancer and infection diseases. Additionally, it has provided improved opportunities for timely diagnosis and prevention of asthma, and treatment of cases refractory to conventional management. Electronic nose (E-nose) is a novel device that includes nano-sensors capable of detecting and identifying specific volatile organic compounds through automated odor recognition (8). E-nose can differentiate the exhaled breath of asthma patients from that of healthy controls and differentiate between the degrees of asthma severity (9). The identification of the phenotype of asthma emphasizes the importance of a personalized approach to the treatment of asthma (10). E-nose can be used to detect patients with eosinophilic, neutrophilic, and paucigranulocytic asthma phenotypes according to their breath prints (11). Nanomedicine can be applied to deliver therapeutic agents for the treatment of asthma, including nano-modified antiasthma drugs and novel nano-drugs (12). Application of nanomedicine to deliver appropriate medications is the matter of discussion and interest already for more than two decades. The use of nanoparticles (NPs) as therapeutics is considered to have several benefits over conventional drugs for the respiratory system (13), including uniform drug distribution, enhanced solubility or dissolution rate, sustained release, macromolecule delivery, and appropriate cell internalization. The benefits of NPs include facilitation of targeted and direct delivery of medications and biological materials where needed. Such an approach with selective 
delivery increases drug concentration in the lungs and reduces its concentration in other parts of the body, which enhances therapeutic pulmonary effects along with lower systemic side effects. Indeed, novel nano-drugs, which are based on new nanoparticles show high efficacy and low toxicity. Another innovative approach in asthma therapy can be based on combining genes with nano-gene carriers. Several preclinical studies have assessed the efficacy of NPs and have shown promising results. Additionally, various NPs, such as liposomes, solid lipid nanoparticles, chitosan, telodendrimers, dendrimers, poly (ethylene imine) (PEI), poly (lactic-co-glycolic acid) (PLGA), and CK30PEG, were developed and suggested for use in asthma therapy.

The bronchodilator salbutamol is used to relieve symptoms and prevent bronchoconstriction. For clinical investigation of a nano-formulation of salbutamol, it was encapsulated into nano-sized non-ionic surfactant-based vesicles, and sustained release of the drug was noted over an 8-hour period after inhalation (14). Additionally, it has been shown that liposomes are suitable form for drug delivery to the respiratory system, as they were able to increase local concentration of salbutamol in the bronchial tree and prolong the duration of therapeutic effect of salbutamol up to ten hours, when compared to the unencapsulated form of salbutamol, in rat and guinea pig models of asthma (15). It is known that high doses of ICS in long-term is associated with numerous systemic side effects, including inhibition of adrenal function, decrease in bone mass and also oral candidiasis. With regard to minimization of the ICS adverse effects, Nasr et al. indicated that PEGylated poly (amidomine) dendrimer could improve drug solubility and increase its pulmonary bioavailability, which would permit dose reduction, lower frequency of administering the drug, as well as reduced toxicity (16). Due to specific structure and characteristics, solid lipid NPs demonstrated good biocompatibility, which was accompanied by the ability to prevent degradation of the incorporated drug, as well as capacity for controlled release. Two solid lipid microparticles (SLMs) chitosan and alginate as mucoadhesive polymers were tested for their biocompatibility and effectiveness by Amore et al. (17) The authors reported that oxidative stress was more effectively controlled by fluticasone propionate (FP)-loaded SLMs than by FP alone. Thus, FP-loaded SLM therapy has potential to improve treatment of chronic inflammatory pulmonary diseases. Some additional substances with anti-inflammatory properties could be prepared as NP formulations and complement the therapy of asthma patients. Wang et al. investigated whether curcumin-solid lipid NPs could reduce hyper-responsiveness in an ovalbumin (OVA)-induced allergic rat model of asthma. The authors found that airway inflammation and hyper-reactivity were suppressed more by curcumin-solid lipid NPs than by curcumin alone (18). Additionally, recombinant Caryota mitis profilin (rCmP)loaded PLGA NPs showed therapeutic benefits by regulating the Th1/Th2 equilibrium in a mouse model of allergic asthma (19). Moreover, well-defined telodendrimers, which are non-toxic nanocarriers, are suitable for pulmonary delivering of dexamethasone in slow-release formulation, with clear anti-inflammatory and anti-allergic effects. Such dexamethasone formulations are superior to simple dexamethasone in the ability to reduce airway hyperresponsiveness (20). The use of bronchodilators and ICSs is considered the main treatment option for asthma. Asthma is a chronic complicated inflammatory lung disease, and there are many potential molecular targets for therapy. Therefore, gene therapy as well as innovative moleculartargeted therapy attract increasing research interest.

Novel NPs in gene therapy for asthma have become new treatment options with regard to effects, such as inhibition of Th2 transcription factors, expression of Th2 cytokines, and function or overexpression of Th2 antagonists. Systems for gene delivery based on synthetic nanoparticles allow adjustable therapeutic gene delivery. NPs can enter cells via cellular uptake and achieve safe and effective targeted gene therapy through interacting with target molecules of the cells. However, gene therapy is still not sufficiently effective since it is challenging to achieve sustained, highlevel transgene expression in the respiratory system due to the need for colloidal stability in physiological fluids as well as the request to overcome the lung's biological barriers. Additionally, the defense mechanisms of the human body might cause rapid degradation of free DNA and RNA. Therefore, NPs, as non-viral nucleic acid delivery vectors, require protection to avoid degradation by nucleases. Several liposomes and macromolecule polymers based nonviral vectors were created to allow conversion of nucleic acids into NPs for pulmonary delivery. It was reported that airway hyper-reactivity and histopathological signs of inflammation can be reduced using chitosan interferonr-DNA NP therapy in an OVA-induced allergic asthma mouse model (21). More recently, Silva et al. used a highly compacted DNA NP system involving plasmid DNA and block copolymers of polyethylene glycol and poly-L-lysine linked by a cysteine residue (CK30PEG) for lung delivery of an anti-inflammatory and anti-fibrotic nanopeptide 
thymulin, in an OVA-induced mouse model of allergic asthma. The instillation of DNA NPs with thymulin plasmids in the murine lung improved lung mechanics by preventing lung inflammation, collagen deposition, and smooth muscle hypertrophy (22). PEI can efficiently form a condense nano-sized complex of negatively charged DNA owing to its high cationic charge density, which protects it from degradation by nucleases. Additionally, PEGylated PEI is able to activate the complement and also to upregulate apoptosis-related genes (23). Thus, biodegradable vectors appear to be important. Highly compacted biodegradable DNA nanoparticles lacking toxicity and demonstrating overall good safety profile after tracheal instillation passed the respiratory epithelium's mucus barrier making them suitable for inhalatory gene therapy of the lungs (24). Moreover, Salem et al. mentioned that a biodegradable NPbased vaccine adjuvant-loaded with cystosine-phosphateguanine, for the management of dust mite allergies can suppress the Th2 asthma response by shifting the immune response to the Th1 type (25). Although several of the above studies have shown the efficacy of administration of nano-formulations involving NPs in the respiratory system, there is a risk of allergic sensitization. The application of NPs in asthma will need careful evaluation.

Asthma is a complex chronic lung disease highly associated with environmental exposure and genetic background, and its pathogenesis involves various cells and cellular processes. There is no specific therapy for asthma owing to its complicated pathogenesis. However, there are several molecules that could be targeted for asthma treatment via NPs, such as cytokines and chemokines, but also various molecules required for silencing or overexpressing certain genes (transcription factors, tyrosine kinases etc.). Recent advances in nanomedicine provide an opportunity to fundamentally improve current therapies for asthma, and the strategy allows delivery of enhanced concentrations of ICSs, which helps reduce systemic adverse effects. Furthermore, it allows delivery of controlled, sustained slow-release compounds with specific absorption characteristics that overcome the issues of mucus and bacterial biofilm burden in asthma patients. Novel NPs provide new hope for gene therapy against asthma in the future. These approaches can improve clinical care in asthma patients. However, nanomedicine is in the early stage in pulmonary care, and additional human data and investment are required to achieve safe and robust nano-therapy. Further improvements and advances in nanotherapy are required to give it more space in clinical practice. The preparation of NPs is an extremely complex process because individual particles cannot be completely controlled. Additionally, multiple analytical and quality control issues exist. The benefits of application of NPs for asthma treatment require more research of different formulation and delivery strategies with the particular emphasis on achieving the desired functionality. We expect that preclinical and clinical studies evaluating smart NPs will help in the development of safe, efficient, nano-based diagnostic and therapeutic platforms for asthma patients, especially those who are resistant to conventional therapy.

\section{Acknowledgments}

None.

\section{Footnote}

Conflicts of Interest: The authors have no conflicts of interest to declare.

Ethical Statement: The authors are accountable for all aspects of the work in ensuring that questions related to the accuracy or integrity of any part of the work are appropriately investigated and resolved.

\section{References}

1. Holgate ST, Wenzel S, Postma DS, et al. Asthma. Nat Rev Dis Primers 2015;1:15025.

2. Raissy HH, Kelly HW, Harkins M, et al. Inhaled corticosteroids in lung diseases. Am J Respir Crit Care Med 2013;187:798-803.

3. Tan LD, Bratt JM, Godor D, et al. Benralizumab: a unique IL-5 inhibitor for severe asthma. J Asthma Allergy 2016;9:71-81.

4. Cooper V, Metcalf L, Versnel J, et al. Patient-reported side effects, concerns and adherence to corticosteroid treatment for asthma, and comparison with physician estimates of side-effect prevalence: a UK-wide, cross-sectional study. NPJ Prim Care Respir Med 2015;25:15026.

5. Adouni Lawani M, Zongo F, Breton MC, et al.Factors associated with adherence to asthma treatment with inhaled corticosteroids: A cross-sectional exploratory study. J Asthma 2018;55:318-29.

6. Pascual RM, Peters SP. Airway remodeling contributes to the progressive loss of lung function in asthma: an overview. J Allergy Clin Immunol 2005;116:477-86; 
quiz 87.

7. Barnes PJ. Severe asthma: advances in current management and future therapy. J Allergy Clin Immunol 2012;129:48-59.

8. Broza YY, Haick H. Nanomaterial-based sensors for detection of disease by volatile organic compounds. Nanomedicine (Lond) 2013;8:785-806.

9. Dragonieri S, Schot R, Mertens BJ, et al. An electronic nose in the discrimination of patients with asthma and controls. J Allergy Clin Immunol 2007;120:856-62.

10. Taylor SL, Leong LE, Choo JM, et al. Inflammatory phenotypes in patients with severe asthma are associated with distinct airway microbiology. J Allergy Clin Immunol 2018;141:94-103.e15.

11. Plaza V, Crespo A, Giner J, et al. Inflammatory Asthma Phenotype Discrimination Using an Electronic Nose Breath Analyzer. J Investig Allergol Clin Immunol 2015;25:431-7.

12. da Silva AL, Cruz FF, Rocco PRM, et al. New perspectives in nanotherapeutics for chronic respiratory diseases. Biophys Rev 2017;9:793-803.

13. Mansour HM, Rhee YS, Wu X. Nanomedicine in pulmonary delivery. Int J Nanomedicine 2009;4:299-319.

14. Bhavna, Ahmad FJ, Mittal G, et al. Nano-salbutamol dry powder inhalation: a new approach for treating broncho-constrictive conditions. Eur J Pharm Biopharm 2009;71:282-91.

15. Chen X, Huang W, Wong BC, et al. Liposomes prolong the therapeutic effect of anti-asthmatic medication via pulmonary delivery. Int J Nanomedicine 2012;7:1139-48.

16. Nasr M, Najlah M, D'Emanuele A, et al. PAMAM dendrimers as aerosol drug nanocarriers for pulmonary delivery via nebulization. Int J Pharm 2014;461:242-50.

17. Amore E, Ferraro M, Manca ML, et al. Mucoadhesive

Cite this article as: Cheng WC, Chen $\mathrm{CH}$. Nanotechnology bring a new hope for asthmatics. Ann Transl Med 2019;7(20):516. doi: $10.21037 /$ atm.2019.09.153 solid lipid microparticles for controlled release of a corticosteroid in the chronic obstructive pulmonary disease treatment. Nanomedicine (Lond) 2017;12:2287-302.

18. Wang W, Zhu R, Xie Q, et al. Enhanced bioavailability and efficiency of curcumin for the treatment of asthma by its formulation in solid lipid nanoparticles. Int $\mathrm{J}$ Nanomedicine 2012;7:3667-77.

19. Xiao X, Zeng X, Zhang X, et al. Effects of Caryota mitis profilin-loaded PLGA nanoparticles in a murine model of allergic asthma. Int J Nanomedicine 2013;8:4553-62.

20. Kenyon NJ, Bratt JM, Lee J, et al. Self-assembling nanoparticles containing dexamethasone as a novel therapy in allergic airways inflammation. PLoS One 2013;8:e77730.

21. Kumar M, Kong X, Behera AK, et al. Chitosan IFNgamma-pDNA Nanoparticle (CIN) Therapy for Allergic Asthma. Genet Vaccines Ther 2003;1:3.

22. Silva LH, da Silva JR, Ferreira GA, et al. Labeling mesenchymal cells with DMSA-coated gold and iron oxide nanoparticles: assessment of biocompatibility and potential applications. J Nanobiotechnology 2016;14:59.

23. Merkel OM, Urbanics R, Bedocs P, et al. In vitro and in vivo complement activation and related anaphylactic effects associated with polyethylenimine and polyethyleniminegraft-poly(ethylene glycol) block copolymers. Biomaterials 2011;32:4936-42.

24. Mastorakos P, da Silva AL, Chisholm J, et al. Highly compacted biodegradable DNA nanoparticles capable of overcoming the mucus barrier for inhaled lung gene therapy. Proc Natl Acad Sci U S A 2015;112:8720-5.

25. Salem AK. A promising CpG adjuvant-loaded nanoparticle-based vaccine for treatment of dust mite allergies. Immunotherapy 2014;6:1161-3. 